\title{
The Temperature Profile for the Innovative Design of the Perforated Fin
}

\author{
Hisham H. Jasim a,b,1 and Mehmet Sait Söylemez ${ }^{a}$ \\ ${ }^{a}$ Department of Mechanical Engineering, University of Gaziantep, 27310 Gaziantep, Turkey \\ $b$ University of Baghdad, Baghdad, Iraq
}

\begin{abstract}
Many studies had proposed the development of the heat sink by using the straight perforated fin .Today increasing the demand for the more effective design due to the growth of the heat sources in the different energy devices. This paper presents the new design (inclined perforated) of the pin fin and find the temperature distribution based on the a novel derivative method. Perforated with rectangular section and different angles of inclination was considered. Signum Function is used for modeling the variable heat transfer area. Set of parameters to handle the conduction and convection area were calculated. Degenerate Hypergeometric Equation (DHE) was used for modeling the complex energy differential equation and then solved by Kummer's series. In the validation process, Ansys 16.0-Steady State Thermal was adopted. For the calculation process, two models (I \& II) were considered. The high agreement of the validation results about $(0.25 \%)$ leads to the big reliability of the presented model. The results show the improvement of the temperature difference and heat transfer ratio according to the increase of the inclination angle and perforated size. Improved of Heat transfer ratio is ranging from $13 \%$ to $50 \%$.
\end{abstract}

Keywords: Inclined perforated, Temperature distribution, Signum function, Degenerate hypergeometric equation, Performance enhancement.

Article History: Received June 12 ${ }^{\text {th }}$ 2016; Received in revised form August $6^{\text {th }}$ 2016; Accepted August 24t 2016 ; Available online

How to Cite This Article: Jasim, H.H and Soylemez, M.S. (2016). The temperature profile for the innovative design of the perforated fin. Int. Journal of Renewable Energy Development, 5(3), 259-266

http://dx.doi.org/10.14710/ijred.5.3.259-266

\section{Introduction}

Development of the modern devices (communications devices, Mechatronics application, control package of the solar cells etc.) depended on the heat sink performance. Also the heat sources can be found in different positions and variable environment, according to the design conditions. For this reason, the researcher tries to find more effective heat sink based on the different techniques like perforated fin. In this field, most studies had changed the operation conditions and geometric shapes for the straight perforation to improve the thermal behavior.

In experimental studies, models with specified geometry were adopted, (Bayram et al. 2008 ${ }^{\text {a }}$ ) studied the variation of the Nusselt Number and the friction factor of the circular perforated based on the change of the parameters (geometric and fluid flow), the efficiency increased by 1.1 and 1.9 based on the inter-fin spacing ratio and clearance ratio.
Saurabh et al. (2014) investigated the influence of the metal on the performance of the circular perforated fin, the experimental tests show the higher heat transfer coefficient at a large number of perforated and a copper material having higher thermal conductivity. (Bayram et al. 2008 ) proved the efficiency can be increased by 1.4 to 2.6 for circular pin fin with a circular section of perforated by optimized the Nusselt number and friction factor separately and then together. (Elshafei 2010) presented the study about the hollow fin with the circular perforated, the results show the increase of the heat transfer depended on the orientation and diameter ratio.

Finite volume is used by the most investigators to solve the governing equations, (Swee et al. 2013) presented the pin fin with the different specification (number and diameter of perforated) to study the recirculation effects. They found the heat transfer increased by $45 \%$, also, the pressure drop of perforated fin decreased by $18 \%$. 
Mohamed et al. (2014) determined the optimum number of pin fins that associated with a minimum value of thermal resistance to improve the heat transfer by use the circular perforated. Monoj et al. (2011) presented the numerical study to determine the heat transfer and pressure drop of elliptical pin fin with straight perforated. Results show the enhancement of heat transfer by $5.6 \%$, a pressure drop improvement by $12 \%$ and the performance of the fin increased by $23 \%$. Ashok et al. (2014) obtained a high increase in convection coefficient about (30\% to $40 \%$ ) for a perforated circular section of pin fin by using the different number of a circular straight perforated. Mohammad et al. (2012) solved The Navier-Stokes and energy equations by the finite volume procedure using the SIMPLE algorithm for the perforation fins. Results show the thermal entrance length is inversely proportional to the number of perforations.

Other investigations found the temperature distribution of perforated fin by analytical study, Kirpikov et al. (1972) reached to the temperature distribution equation of perforated flat plate based on the mathematical model that solved by Fourier series and Flocke's theory. Zan WU et al. (2012) evaluated the performance of the isolated isothermal perforation plates with Staggered pattern. The results show the increase of the total heat transfer rate depended on the effects of the open area ratio and inclined angle.

All recent studies, used the straight perforation at different geometries and various operation conditions to improve the performance of the fin. New approach introduces through this article. Two points of novelty propose in this study, firstly at geometric design, the inclined perforated with rectangular section is considered. Secondly, at analytical process, the new differential technique is used to derive the general form of the temperature distribution and regardless of the perforated shape.

\section{Geometric Model}

The inclined perforated of the pin fin is shown in Figure 1. In the general form, an undefined section of perforated for the pin fin was considered. Inclination started from $(\beta=0)$ at straight perforated.

The fin has a rectangular cross section area. Also, the base fin located at the $x-y$ plane and the fin length at y-axis. According to above geometry, the heat transfer area is changing with y-direction and inclination angle

\section{Analytical solution}

\subsection{Energy analysis and assumption}

Applied Energy balance to the element shown in Figure 2, to obtain the energy equation of the perforated region.
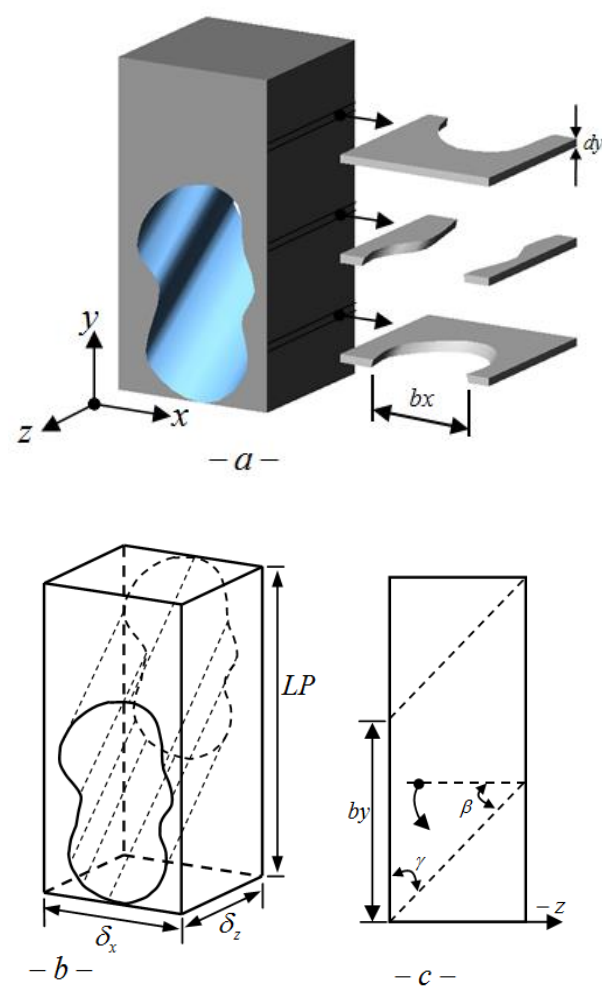

Fig. 1 Fin with inclined perforated, a- 3-D model with different layer, b- Illustration diagram, c- Side view.

. Heat transfer analysis for inclined perforated region relies on a set of assumptions:

1- Impairment value of the Biot number at $\mathrm{z}$-axis and $\mathrm{x}$-axis leads to the one-dimensional heat transfer.

2- Steady heat conduction with no heat generation.

3- Constant conductivity.

4- Constant base temperature.

5- Insulation tip fin.

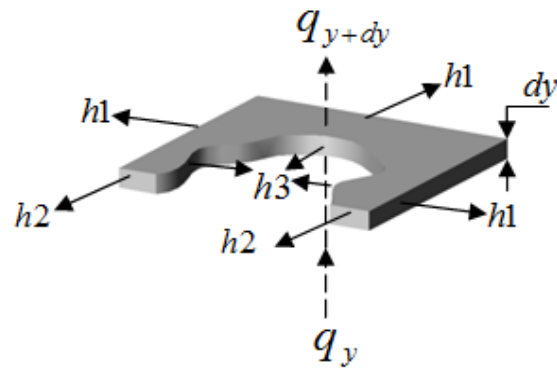

Fig. 2 Element description

6- Radiation effects are neglected. Uniform ambient temperature and convection heat 
transfer coefficient assumed to be uniform over all surfaces of the fin.

7- Convection coefficient is divided into three types (external non-perforated (h1), external perforated (h2) and internal perforated (h3)).

Based on these assumptions, the energy equation can be written as shown in equation (1)

$$
\begin{aligned}
\frac{d}{d y}\left(A_{\text {cond. }} \frac{d \theta}{d y}\right) d y= & {\left[h 1 P_{\text {conv } 1}+h 2 P_{\text {conv } 2}\right.} \\
& \left.+h 3 P_{\text {conv } 3}\right] \frac{\theta d y}{k}
\end{aligned}
$$

Where:

$$
\begin{array}{ll}
\theta & =\text { Temperature difference } T(y)-T_{\infty} \\
h 1,2,3 & =\text { Convection coefficients }\left[\mathrm{W} / \mathrm{m}^{2} \mathrm{~K}\right] \\
P_{\text {conv } 1,2,3} & =\text { Perimeter according to the } h 1,2,3[\mathrm{~m}] \\
A_{\text {cond }} & =\text { conduction area }\left[\mathrm{m}^{2}\right] \\
k & =\text { Thermal Conductivity }[\mathrm{W} / \mathrm{m} \mathrm{K}]
\end{array}
$$

\subsection{General solution}

Expanded conduction heat transfer term of Equation (1) and substitution $\theta=G / \sqrt{A_{\text {cond }}}$ leads to the linear second order differential equation with a variable coefficient.

$$
\frac{d^{2} G}{d y^{2}}+\lambda(y) G=0
$$

Where:

$$
\begin{aligned}
& \lambda(y)=-\left[\frac{A_{\text {cond. }}^{\prime}{ }^{2}}{4 A_{\text {cond. }}^{2}}+\frac{A_{\text {cond. }}^{\prime \prime}}{2 A_{\text {cond. }}}+\frac{h 1 P_{\text {conv } 1}}{k A_{\text {cond } .}}\right. \\
&\left.+\frac{h 2 P_{\text {conv } 2}}{k A_{\text {cond. }}}+\frac{h 3 P_{\text {conv } 3}}{k A_{\text {cond } .}}\right] \\
& A^{\prime} \quad=\text { First derivative of area } \\
& A^{\prime \prime} \quad \text { Second derivative of area }
\end{aligned}
$$

Equation (2) placed in another form by fitting $\lambda(y)$ using polynomial function with coefficients $(p 1, p 2, p 3)$ and substitution:

$$
\begin{aligned}
& \xi=y+(p 2 / 2 p 1) \& d 1=p 3-\left(p 2^{2} / 4 p 1\right) \\
& \frac{d^{2} G}{d \xi^{2}}-\left(p 1 \xi^{2}+d 1\right) G=0
\end{aligned}
$$

Can be found Degenerate Hypergeometric Equation (DHE) from Equation (3) by assuming $\vartheta=\xi^{2} \sqrt{p 1}$ ,$u=e^{\vartheta / 2} G$. (Andrei et al. 2003)

$$
\vartheta \frac{d^{2} u}{d \vartheta^{2}}+(k u 1-\vartheta) \frac{d u}{d \vartheta}-(k u 2) u=0
$$

Where:

$$
k u 1=\frac{1}{2}, \quad k u 2=\frac{1}{4}\left(\frac{d 1}{\sqrt{p 1}}+1\right)
$$

According to kummer's series (Hazewinkel 1995), the general solution of (DHE) is:

$$
\begin{gathered}
u(\vartheta)=C O_{i} \phi(k u 1, k u 2, \vartheta)+ \\
C O_{i+1} \psi(k u 1, k u 2, \vartheta) \\
, i=1,3,5, \ldots .
\end{gathered}
$$

Where:

$\phi=$ confluent hypergeometric function of the first kind. $\psi=$ confluent hypergeometric function of the second kind.

$C O=$ Constant for the general solution

Boundary conditions, according to Equation (5):

$$
\left.u\right|_{\vartheta=\frac{p 2^{2}}{4 p 1^{3 / 2}}}=\theta_{b} \sqrt{A_{b}} e^{\frac{p 2^{2}}{8 p 1^{3 / 2}}},\left.\frac{d u}{d \vartheta}\right|_{\vartheta=\left(L P+\frac{p 2}{2 p 1}\right)^{2} \sqrt{p 1}}=0
$$

The boundary conditions at original form can be written as below:

$$
\left.\theta\right|_{y=0}=\theta_{b} \quad,\left.\quad \frac{d \theta}{d y}\right|_{y=L P}=0
$$

Equation (5) solved based on the boundary conditions. The general solution can be obtained after rewriting the parameters by re-compensation to all substitutions in original form.

$$
\begin{aligned}
\frac{\theta(y)}{\theta_{b}}= & \frac{e^{\frac{-\left(y+\frac{p 2}{2 p 1}\right)^{2} \sqrt{p 1}}{2}}}{\sqrt{A_{\text {cond }}}} \frac{e^{\frac{p 2^{2}}{8 p 1^{3 / 2}}} \sqrt{A_{b}}}{\zeta 1-\zeta 2}[\zeta 3 \\
& \phi\left(k u 1, k u 2,\left(\left(y+\frac{p 2}{2 p 1}\right)^{2} \sqrt{p 1}\right)\right) \\
& \left.-\zeta 4 \psi\left(k u 1, k u 2,\left(\left(y+\frac{p 2}{2 p 1}\right)^{2} \sqrt{p 1}\right)\right)\right]
\end{aligned}
$$


Where:

$A_{b}=$ base area

$\theta_{b}=$ temperature at fin base

$\zeta 1=\left[\zeta 3 \phi\left(k u 1, k u 2, \frac{p 2^{2}}{8 p 1^{3 / 2}}\right)\right]$

$\zeta 2=\left[\zeta 4 \psi\left(k u 1, k u 2, \frac{p 2^{2}}{8 p 1^{3 / 2}}\right)\right]$

$\zeta 3=\psi^{\prime}\left[k u 1, k u 2,\left(\left(L P+\frac{p 2}{2 p 1}\right)^{2} \sqrt{p 1}\right)\right]$

$\zeta 4=\phi^{\prime}\left[k u 1, k u 2,\left(\left(L P+\frac{p 2}{2 p 1}\right)^{2} \sqrt{p 1}\right)\right]$

\section{Inclined perforated with rectangular section}

Fin with inclined perforated is classified into three regions (solid, inclined perforated and then solid) as illustrated in Figure 3. Conduction area ( $A_{\text {cond. }}$ ) and convection perimeter $\left(P_{\text {conv. }}\right)$ is used for modeling of the heat transfer area. Change of the inclination angle leads to different forms of the heat transfer area as shown in figure 3 for two main cases. ( $P_{\text {conv. }}$ ) divided into three regions [external non-perforated, external perforated and internal perforated] based on the classification of convection coefficient. In the mathematics, Signum function ( $s g n$ ) is used for modeling the different function (John et al. 1998).

For this study, ( $s g n$ )used to represent the opposite approach of variables $\left(A_{\text {cond }}\right)$ and $\left(P_{\text {conv. }}\right)$ with the y-axis as below:

$$
\begin{aligned}
A_{\text {cond } .}= & \frac{\delta_{z} b x}{a}\left(a_{1}-y\right) \operatorname{sgn}(a-y)+ \\
& \left(\delta_{z} \delta_{x}\right)-\delta_{z} b x \\
P_{\text {conv } 1}= & 2 \delta_{z}+a_{2} \\
P_{\text {conv } 2} & =\delta_{x}-b x \\
P_{\text {conv } 3}= & \frac{-2 \delta_{z}-b x}{a}\left(a_{1}-y\right) \operatorname{sgn}(a-y) \\
& +2 \delta_{z}+b x
\end{aligned}
$$

Where:

$$
\operatorname{sgn}(a-y)=\tanh [(N(a-y)], \quad N>>1
$$

Extreme ends of the $\left(A_{\text {cond. }}\right)$ and $\left(P_{\text {conv. }}\right)$ can be represented by a point or by a line depended on the inclined angle. A group of variables $\left(a, a_{1}, a_{2}\right)$ was calculated to find the heat transfer area at any specification as shown in the table (1).
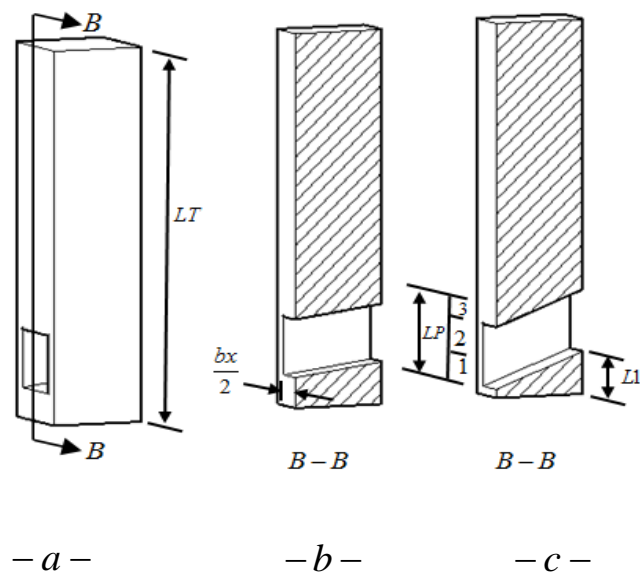

Fig. 3 Rectangular perforated with different angle a) 3-D plot $b$ ) smaller angle $c$ ) bigger angle

Table (1)

Constant Value

\begin{tabular}{cccc}
\hline \multirow{2}{*}{ Region No. } & \multicolumn{3}{c}{ Constant value } \\
\cline { 2 - 4 } & $\mathrm{a}$ & $\mathrm{a} 1$ & $\mathrm{a}_{2}$ \\
\hline 1 & $\mathrm{~L} 11$ & $\mathrm{~L} 11$ & $\delta_{x}$ \\
2 & $\mathrm{~L} 11$ & $\mathrm{y}$ & 0 \\
3 & $\mathrm{~L} 11$ & $\mathrm{~L} 22$ & $\delta_{x}$ \\
& $L 11=\delta_{z} \tan \beta \quad, L 22=L P-L 11$ \\
\hline
\end{tabular}

The solid region was solved based on the (Incropera et al. 2007), while the perforated region was solved based on the equation (6). Matlab (R2014a) language was used to find the results according to heat transfer by natural convection and Rayleigh number $\left(\mathrm{Ra}=10^{6}\right)$. In this study, two models (I and II) were adopted as described below: $\delta_{x}=\delta_{z}=0.01(\mathrm{~m}), b x=b y=0.007(\mathrm{~m})$

$$
\begin{gathered}
, L T=0.05(\mathrm{~m}) \quad \text { Model I } \\
\delta_{x}=\delta_{z}=0.01(\mathrm{~m}), b x=b y=0.008(\mathrm{~m}) \\
, L T=0.05(\mathrm{~m}) \quad \text { Model II }
\end{gathered}
$$

\section{Coefficient of the convection heat transfer}

Various convection heat transfer coefficients were appeared due to inclined perforated. Moreover, Convection coefficients depended on the properties of 
cooling fluid, specifications of the perforated fin and the open perforated ratio (ROP). ROP represents the ratio of actual perforated area to maximum perforation effects.

Empirical correlations from (Incropera et al. 2007; Zan et al. 2012) were used to find the convection coefficients $\mathrm{h}_{1}$ and $\mathrm{h}_{2}$ respectively. While the correlation from (Raithby et al. 1998) was modified to become useful for calculated the $\mathrm{h}_{3}$ that is used for the inclined perforated.

$N u=\frac{h 1 L T}{k_{\text {air }}}=\left\{0.825+\frac{0.387 R a^{1 / 6}}{\left[1+\left(\frac{0.492}{\mathrm{Pr}}\right)^{9 / 16}\right]^{8 / 27}}\right\}^{2}$

Where:

$$
\begin{aligned}
& R a=\frac{g \frac{1}{T_{f}}\left(\bar{T}-T_{\infty}\right) L P^{3}}{v^{2}} \operatorname{Pr}, \quad T_{f}=\left(\bar{T}+T_{\infty}\right) / 2 \\
& k_{\text {air }}=\text { thermal conductivity of the air }
\end{aligned}
$$

$$
\begin{gathered}
h 2=(1+0.75 R O P) h 1 \\
N u=\frac{h 3 L c}{k_{\text {air }}}=\left(\left(\frac{R a}{14.225}\right)^{-1.5}+\right. \\
\left.\left[\frac{0.81 R a^{0.25}}{\left[1+\left(\frac{0.492}{\operatorname{Pr}}\right)^{9 / 16}\right]^{4 / 9}}\right]^{-1.5}\right)^{1 /-1.5}
\end{gathered}
$$

Where:

$$
\begin{aligned}
& R a=\frac{g(\sin \gamma) \frac{1}{T_{f}}\left(\bar{T}-T_{\infty}\right) L c^{4}}{v^{2} \delta_{z}} \operatorname{Pr} \\
& L c=\frac{b x b y}{b y+b x \cos \beta} \\
& g=\text { Gravity }\left[\mathrm{m} / \mathrm{s}^{2}\right] \\
& U=\text { Kinematic viscosity }\left[\mathrm{m} / \mathrm{s}^{2}\right]
\end{aligned}
$$

\section{Numerical solution and simulation}

Thermal analysis have been simulated using Ansys 16.0-Steady State Thermal for the models I and II. For each case, several grids are studied to ensure that the solutions are independent grids and the results are shown in table (2). The minimum temperature was

\begin{tabular}{|c|c|c|c|}
\hline \multicolumn{2}{|c|}{ Inclination angle } & Grid & $\begin{array}{c}\text { Min. } \\
\text { Temperature }\end{array}$ \\
\hline \multirow{12}{*}{$\begin{array}{l}\overline{0} \\
\frac{0}{0} \\
\Sigma\end{array}$} & \multirow[t]{4}{*}{$\beta=8$} & 312544 & 87.1 \\
\hline & & 380965 & 87.69 \\
\hline & & 400879 & 87.903 \\
\hline & & 415312 & 87.904 \\
\hline & \multirow[t]{4}{*}{$\beta=15$} & 365786 & 87.05 \\
\hline & & 410324 & 87.34 \\
\hline & & 440751 & 87.88 \\
\hline & & 466414 & 87.89 \\
\hline & \multirow[t]{4}{*}{$\beta=22$} & 590711 & 87.03 \\
\hline & & 650236 & 87.52 \\
\hline & & 688239 & 87.87 \\
\hline & & 703567 & 87.88 \\
\hline \multirow{12}{*}{$\begin{array}{l}\equiv \\
\frac{0}{0} \\
\sum \\
\Sigma\end{array}$} & \multirow[t]{4}{*}{$\beta=9$} & 392782 & 86.754 \\
\hline & & 410231 & 86.881 \\
\hline & & 440987 & 87.134 \\
\hline & & 479486 & 87.136 \\
\hline & \multirow[t]{4}{*}{$\beta=17$} & 791286 & 86.882 \\
\hline & & 813054 & 86.901 \\
\hline & & 851200 & 87.109 \\
\hline & & 877123 & 87.111 \\
\hline & \multirow[t]{4}{*}{$\beta=25$} & 981570 & 86.682 \\
\hline & & 990540 & 86.891 \\
\hline & & 1001239 & 87.072 \\
\hline & & 1127243 & 87.076 \\
\hline
\end{tabular}
compared until independent grids were succeeded. Some of grids configurations are illustrated in fig. (4). The material of the fin is aluminum with constant thermal conductivity ( $k=222 w / m . K$, (A1050)). All the results are calculated at the base temperature $\left(90^{\circ} \mathrm{C}\right)$ and air temperature $\left(25^{\circ} \mathrm{C}\right)$.

\section{Table 2}

Grid independent studies

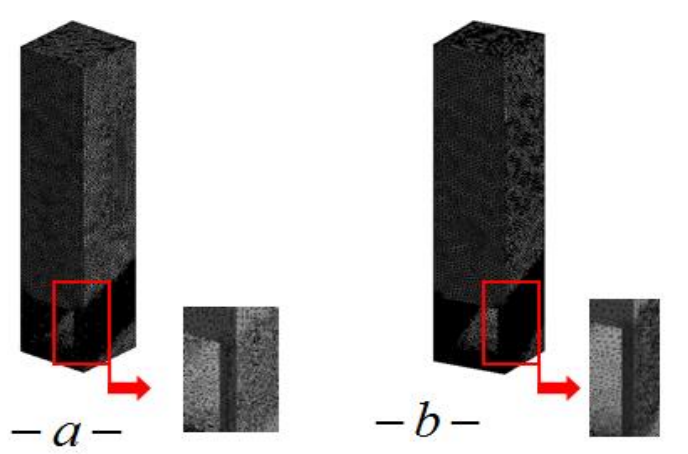

Fig. 4 grid configuration for inclined perforated a) Model I $\beta=22$ b)Model II $\beta=25$

\section{Results and discussion}

\subsection{Validation of analytical model}

In the validation process, three inclination angles were considered. The distribution of the fin temperature $\left(\theta / \theta_{b}\right)$ was calculated along the fin length $(\mathrm{y} / \mathrm{LT})$ to show the convergence between results. Figures (5\&6) are illustrating the temperature distribution of the Model I and Model II, respectively. All figures contain the temperature contours that are obtained from the simulation work. Increase of the inclination angle leads to growth the convection area and decrease in the conduction area. Also the increased of the perforated size can be lead to the same improvement in the convection area. 
Citation: Jasim, H.H and Soylemez, M.S. (2016). The temperature profile for the innovative design of the perforated fin. Int. Journal of Renewable Energy Development, 5(3), 259-266, doi: 10.14710/ijred.5.3.259-266

P a g e | 264

Increase of the convection effects leads to big drop of the fin temperature through the perforated region. Agreements between results are starting from complete resemblance to a maximum difference of $(0.25 \%)$.

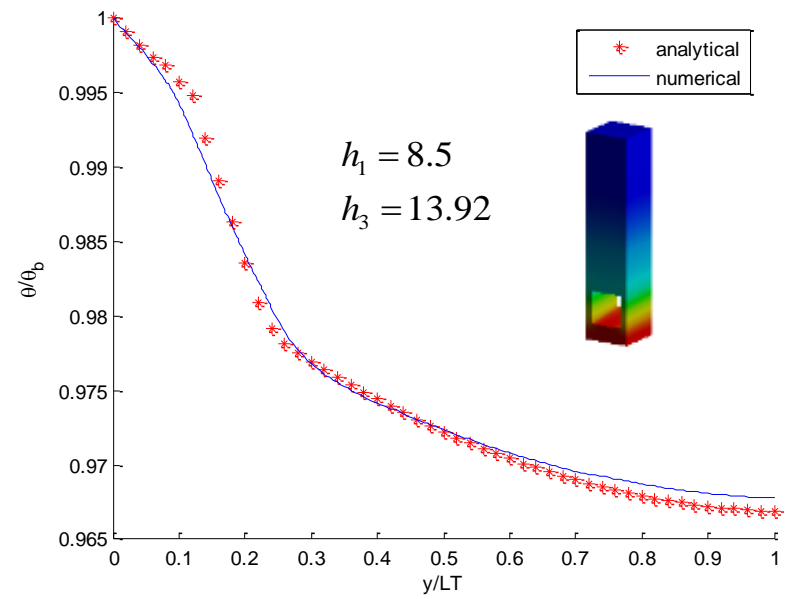

$-a-$

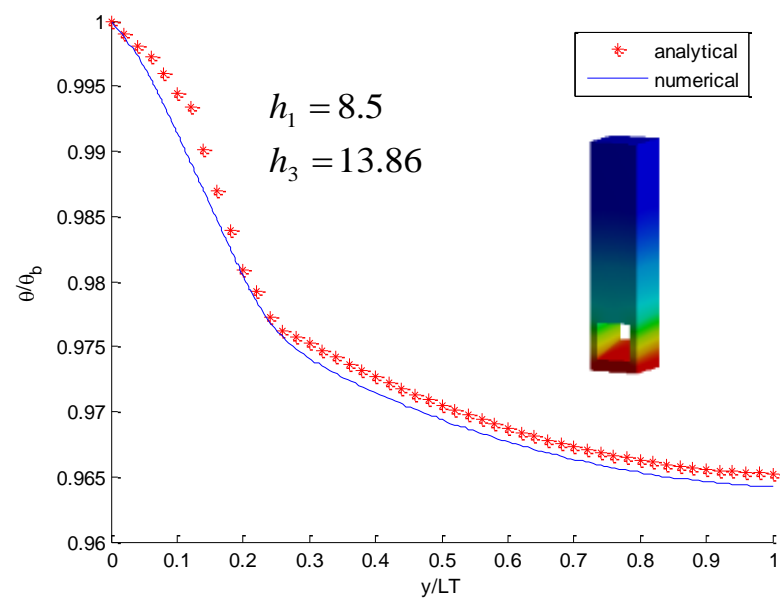

$-b-$

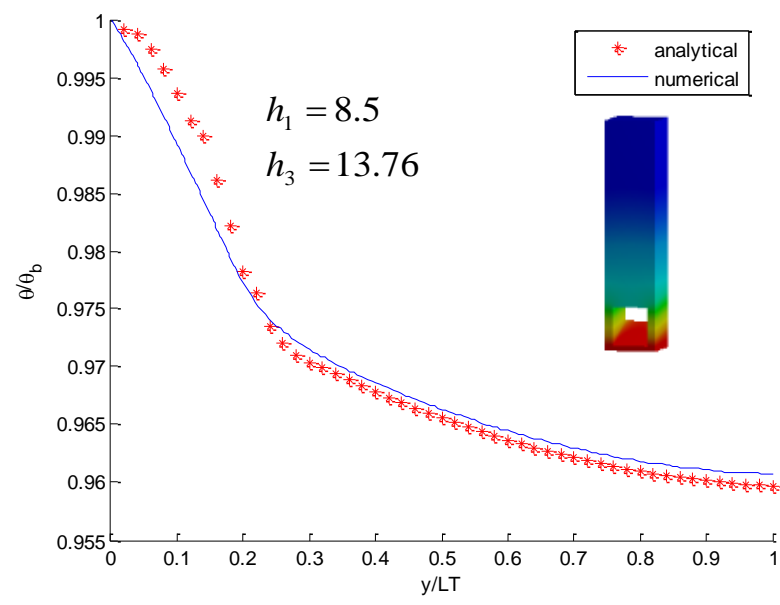

$-c-$

Fig. 5 Comparisons between analytical and numerical results for a) $\beta=8^{\circ}$ b) $\beta=15^{\circ}$ c) $\beta=22^{\circ}$

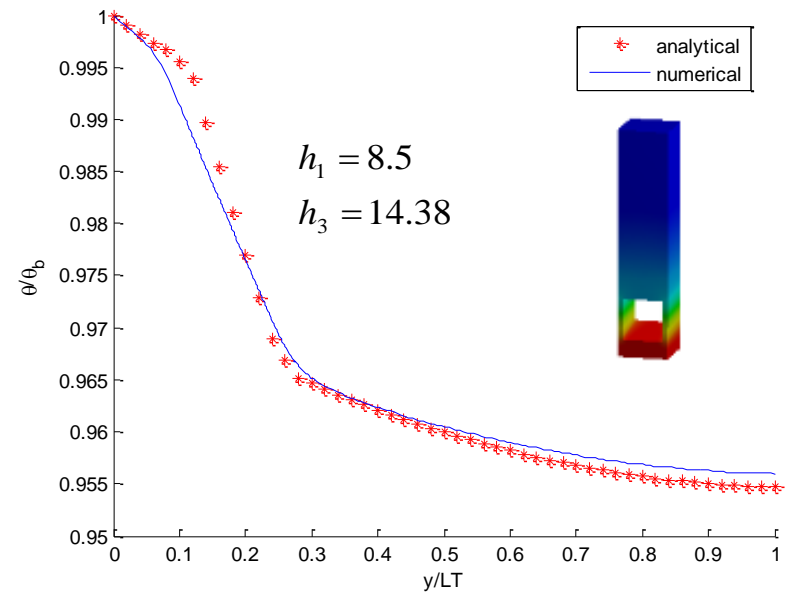

$-a-$

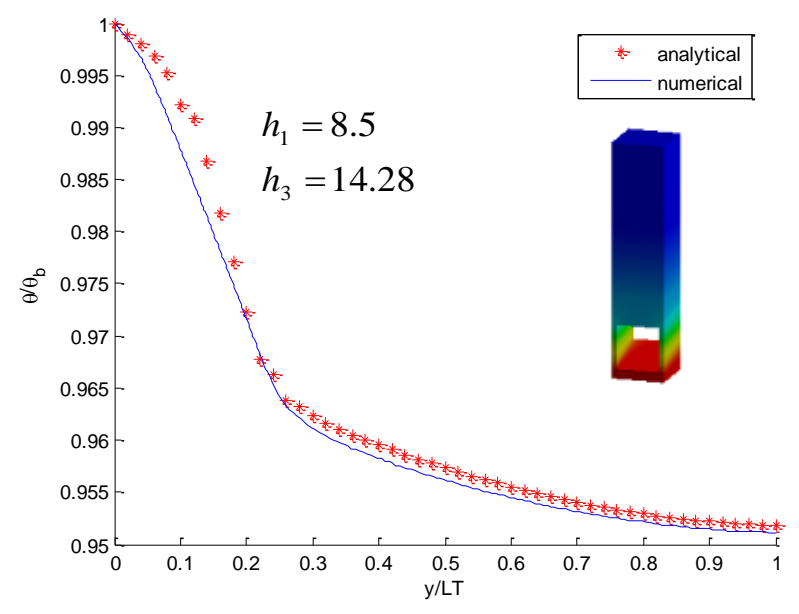

$-b-$

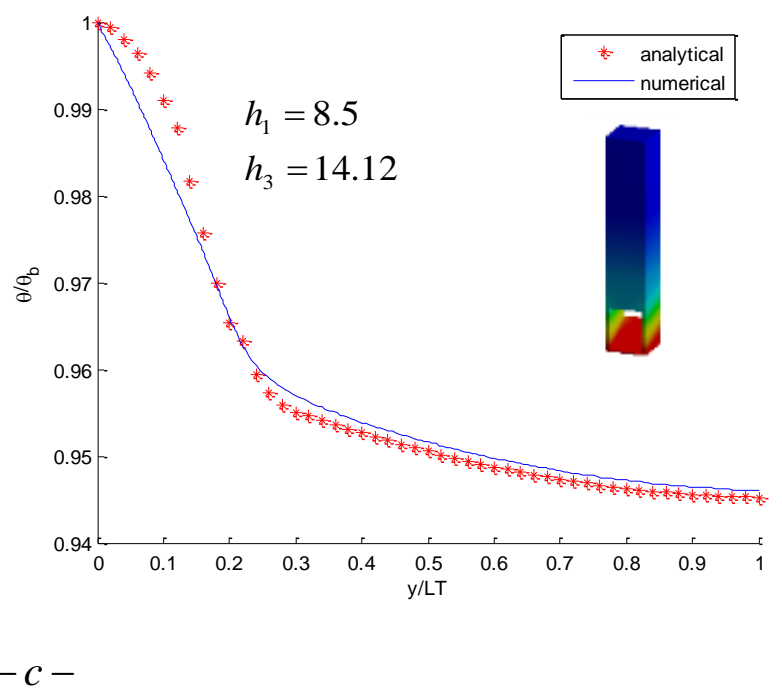

Fig. 6 Comparisons between analytical and numerical results for a) $\beta=9^{\circ}$ b) $\beta=17^{\circ}$ c) $\beta=25^{\circ}$ 


\subsection{Effects of inclination angle (temperature)}

Figures (7 and 8) show the temperature distributions $\left(\theta / \theta_{b}\right)$ for the model I and model II respectively. The results show the increase of the inclination leads to the improvement of the thermal performance, according to the decrease of the temperature difference.

When using the inclined perforated, Multi advantages can be happened:

- Increase of the inner convection area

- Distribute the inner convection area on the longer length in the y-axis.

- Increase of the external perforated area.

- Development of the recirculations in y-z as well as the $\mathrm{X}$-z planes).

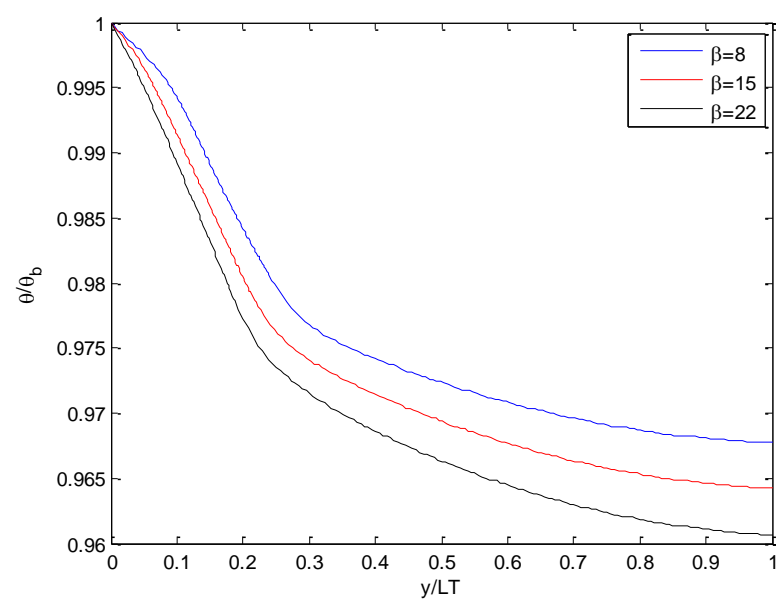

Fig. 7 Variation of temperature distribution with various inclination angles .

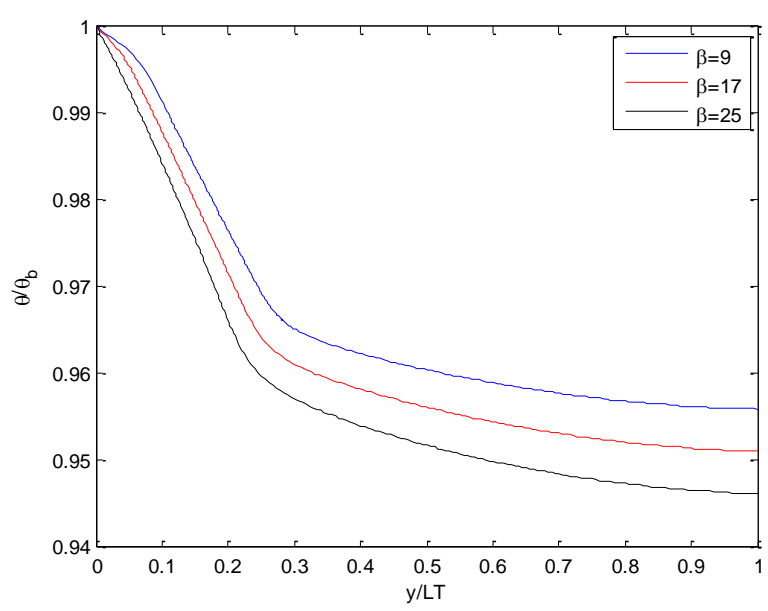

Fig. 8 Variation of temperature distribution with various inclination angles .

\subsection{Effects of inclination (heat ratio)}

Heat transfer is possible to increase, according to the improvement of the temperature distributions and convection heat transfer coefficients. The heat transfer ratio $\left(q_{p} / q_{s}\right)$ is described as the ratio between the heat transfer of a perforated fin to heat transfer of the solid fin at the same properties.

$q_{p \text { or } s}=-\left.k A_{b} \frac{d \theta}{d y}\right|_{y=0}$

Figures ( 9 and 10) illustrate the heat transfer ratio for the models I and II, respectively. Enhanced of the heat transfer due to the increase of the inclination angle. The reason for that, increase the slope of the temperature curve with greater angles. Improved of Heat transfer ratio about (13\%-44\%) for the model I, and (33\%-50\%) for model II.

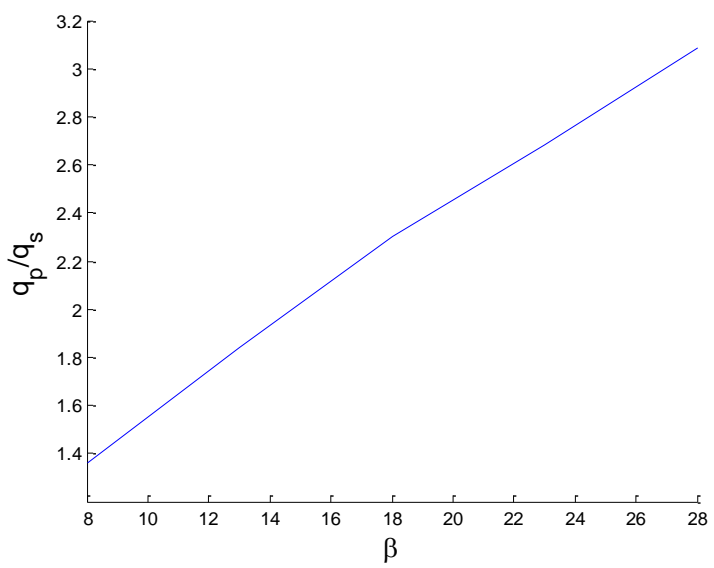

Fig. 9 effects of inclination angles on the heat transfer.

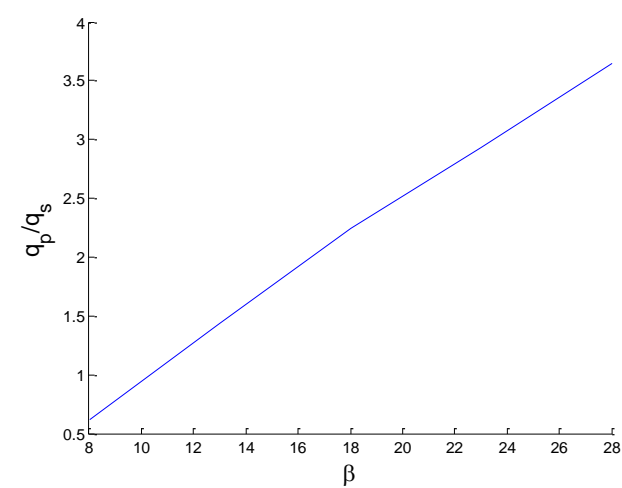

Fig. 10 effects of inclination angles on the heat transfer.

\section{Conclusion}

In addition to the new geometry (inclined perforated), this paper presented a novel technique at 
two major points, modeling of the variable heat transfer area based on Signum function and solves energy differential equation for complex geometry by (DHE). The high agreement of the validation results leads to being sure; the present mathematic model has big reliability. In this study, the use of the common perforated shapes (rectangular) showed the accuracy level and flexibility of the mathematic model. Consequently, general solution for present study taken as the basis to resolve any inclined perforation shape based on modeling of the area. Fin performance can be improved based on the advantages of inclined perforated. Increasing of the inclination angle leads to enhance the temperature distribution and heat transfer, on the other hand, all previous advantages could be better if the perforated size was increased.

Abbreviations
DHE : Degenerate Hypergeometric Equation
ROP
sgn : Open perforated ratio

\section{References:}

Andrei D. Polyanin and Valentin F. Zaitsev, 2003 . hand book of Exact solution for ordinary differential equations, $2^{\text {nd }}$ ed (USA). By CHAPMAN \& HALL/CRC , P.213-490.

Ashok Fule, A .M Salwe , A Zahir Sheikh, and Nikhil Wasnik, (2014). convective heat transfer comparison between solid and perforated pin fin, international journal of mechanical and robotics research,3(2).

Bayram Sahin, and Alparslan Demir, (2008)a . Performance analysis of a heat exchanger having perforated square fins, Applied Thermal Engineering, 28, 621-632.
Bayram Sahin, and Alparslan Demir, (2008)b. Thermal performance analysis and optimum design parameters of heat exchanger having perforated pin fins , Energy Conversion and Management,49,16841695.

Elshafei .E. A. M, (2010). Natural Convection Heat Transfer from a Heat Sink with Hollow / Perforated Circular Pin Fins, Energy, 35,28702877.

Hazewinkel. M, (1995). Encyclopaedia of Mathematics: A-Integral Coordinates, Springer science and business media, [p:105-110 and 797-800].

Incropera, Dewitt, Bergman and Lavine , 2007. Fundamental of heat and mass transfer, John Wiley \& Sons; 6th edition , [p:95-160 and 560-594].

John W. Harris and Horst Stocker , 1998. Hand book of mathematics and computational science, springer (USA) [p:130-150].

Kirpikov.V.A and Leifman.I.I, 1972. calculation of the temperature profile of a perforated fin, instituted of chemical apparatus design, Moscow. 123(2),316-321

Mohamed L. Elsayed, and Osama Mesalhy ,(2014). Studying the performance of solid/perforated pin-fin heat sinks using entropy generation minimization, Heat Mass Transfer, [10.1007/s00231014-1451-9].

Mohammad Reza Shaeri, Tien-Chien Jen, (2012). The effects of perforation sizes on laminar heat transfer characteristics of an array of perforated fins, Energy Conversion and Management 64 (2012) 328-334.

Monoj Baruah, Anupam Dewan and P. Mahanta, (2011). Performance of Elliptical Pin Fin Heat Exchanger with Three Elliptical Perforations, CFD Letters Vol. 3(2), [S2180-1363(11)3265-X].

Raithby GD, and Hollands KGT,1998. Natural convection. In: Warren M. Rohsenow, James R Hartnett, Young I. Cho, Handbook of heat transfer, MCGRAW-HILL $3^{\text {rd }}$ edition, [p:4-1 to 4-80].

Saurabh D. Bahadure, and Gosavi , 2014. Enhancement of Natural Convection Heat Transfer from Perforated Fin, International Journal of Engineering research, 3(9), 531-535.

Swee-Boon Chin, Ji-Jinn Foo, and Yin-Ling La , 2013. Forced convective heat transfer enhancement with perforated pin fins, Heat Mass Transfer, 49,1447-1458.

Zan WU, Wei LI, Zhi-jian SUN , and Rong-hua HONG, 2012. Modeling natural convection heat transfer from perforated plates, Journal of Zhejiang Unversity-SCIENCE A [13(5):353-360]. 